Stanton, N. A. \& Pinto, M. (2000) Behavioural compensation by drivers of a simulator when using a vision enhancement system. Ergonomics 43, 1359-1370.

\title{
Behavioural Compensation by Drivers of a Simulator when using a Vision Enhancement System
}

\author{
Neville A. Stanton and Marcel Pinto \\ Department of Design \\ Brunel University \\ Egham \\ Surrey \\ TW20 0JZ \\ UK
}

Technological progress is suggesting dramatic changes to the tasks of the driver, with the general aim of making the driving environment safer. Before any of these technologies are implemented, empirical research is required to establish if these devices do, in fact, bring about the anticipated improvements. Initially, at least, simulated driving environments offer a means of conducting this research. The study reported in this paper concentrates on the application of a vision enhancement system within the risk homeostasis paradigm. It was anticipated, in line with RHT, that the drivers would compensate for the reduction in risk by increasing their speed. The results of this study support the hypothesis although, after a simulated failure of the vision enhancement system, drivers did reduce their speed due to reduced confidence in reliability of the system.

KEYWORDS: Vision enhancement, driving, RHT, simulation 
Stanton, N. A. \& Pinto, M. (2000) Behavioural compensation by drivers of a simulator when using a vision enhancement system. Ergonomics 43, 1359-1370.

\section{Vision enhancement for drivers of vehicles}

There has been substantial technological progress regarding vehicle technology in recent years. Stanton and Young (1998) present a dichotomy of systems: i.e. those that replace the driver and those that support the driver. Research into systems that replace the driver seems, such as adaptive cruise control, to suggest that vehicle manufacturers should proceed with caution (Nilsson 1995, Stanton \& Marsden 1996, Stanton et al. 1997, Stanton \& Young 1998, Young \& Stanton 1997). Research on systems that support the driver, such as navigational aids (Dingus et al. 1997a, Jackson 1998), route guidance (Srinivasan \& Jovanis 1997) and collision warning devices (Dingus et al. 1997b), present a more optimistic picture. The marked difference between these dichotomous technologies, is that the former may keep the driver out of the vehicle control loop until too late, whilst the latter set support tasks that the driver undertakes whilst still controlling the vehicle.

Vision Enhancement systems work by employing an infrared camera next to the headlights at the front of the car. The road view captured by the camera is then projected to provide a head-up display to the driver, overlaid on the real world. This image enables drivers to see the road and objects in their path that otherwise might be missed due to poor illumination. The system enhances night-time vision where objects may be dull even with ordinary headlight illumination. The benefits of Vision Enhancement become even more obvious when driving in foggy conditions. Under such conditions the driver's range of vision may be reduced to only a few metres. With the Vision Enhancement system activated the road view becomes greatly enhanced, enabling the driver to literally see through the fog. As an illustration, figures 1 and 2 show the road view without (figure 1) and with (figure 2) the vision enhancement system in a driving simulator.

FIGURES ONE ABOUT HERE PLEASE

Figure 1. Driving in fog without vision enhancement.

FIGURES TWO ABOUT HERE PLEASE

Figure 2. Driving in fog with vision enhancement. 
Stanton, N. A. \& Pinto, M. (2000) Behavioural compensation by drivers of a simulator when using a vision enhancement system. Ergonomics 43, 1359-1370.

With the Vision Enhancement system activated, the road view becomes greatly enhanced, enabling the driver to literally see through the fog. It has been suggested that under some circumstances, the stress associated with driving can lead drivers to simple, inappropriate, actions when faced with complex, mentally straining, situations (Hoyos, 1988). Under these circumstances, vision enhancement systems could assist the driver by reducing the demands made upon them.

\section{Risk Homeostasis Theory}

It is the controversial assertion of Risk Homeostasis Theory (RHT: Wilde 1982, 1994) that the unique determinant of accident loss (all costs associated with accidents) is not the intrinsic risk within the environment, rather it is the target level of risk desired by individuals within the environment. Determinants of individuals' target levels of risk are indicated by the optimization of the utilities presented in box (a) in figure 3 . If one or more of the utilities in box (a) change, then the target level of risk will change, and ultimately this will affect accident loss. RHT was originally proposed as a population level, closed loop, description of driver behaviour. Information entering the closed loop would include that about vehicle accidents (e.g. reading about accidents, being involved in an accident or a near miss, etc.). This information would warn the population about actual levels of risk in the environment. If target risk and actual risk are unbalanced, then RHT asserts that individuals will change their behaviour to bring the two into line. RHT has three main pathways that individuals can use to compensate for changes in target risk: behavioural adjustments within the environment, mode migration and avoidance.

\section{FIGURE THREE ABOUT HERE}

\section{Figure 3. Risk homeostasis theory}

Behavioural adjustments: there may be numerous ways that individuals can change the level of risk through behavioural adjustments, e.g. in driving: more or less overtakes, drive faster or slower, increase or decrease attention, more frequent or less frequent mirror checks.

Mode migration: if individuals cannot reduce the level of risk by behavioural adjustments, they could use a safer form of transport, e.g. the train.

Avoidance: an alternative pathway is simply to avoid the situation if it is perceived as too risky, e.g. not to make a particular journey because of adverse weather conditions. 
Stanton, N. A. \& Pinto, M. (2000) Behavioural compensation by drivers of a simulator when using a vision enhancement system. Ergonomics 43, 1359-1370.

In the driving context (with which RHT is normally associated) a driver may interpret snow on the road as an indication of an increased level of intrinsic risk in the environment. To compensate for this (i.e. to restore the previously existing level of risk) the driver may decide to drive slower, leave a greater gap for the vehicle in front, and so on. However, if the driver perceives that, no matter what behavioural adjustments are made, it is impossible to restore the previous level of risk, then an alternative mode of transport may be chosen (mode migration). If this alternative form of transportation is still too risky, then the driver may decide not to travel under the prevailing conditions (avoidance). RHT is not without its critics, however. McKenna (1988), for example, argues that the level of risk people are willing to accept is not the sole determinant in accident involvement. Others, such as Trimpop (1996), argue that the weight of evidence tips in favour of RHT.

Proponents of RHT would predict that the main benefits (in terms of accident loss) are to be gained by directly manipulating the utilities (for example, increasing the costs and decreasing the benefits associated with risky behaviours and decreasing the costs and increasing the benefits associated with cautious behaviours) rather than attempting to adjust intrinsic risk, which will only be compensated for in any case. If drivers really do act in such a manner, this might not lead to the expected benefits arising from a car fitted with vision enhancement system. Without a vision enhancement system, drivers are forced to slow down in conditions of poor visibility in order to drive safely. Conversely, with a vision enhancement system engaged, the driver's view of the road scene ahead would not be so adversely impaired and the driver may perceive this as a much less risky situation. The driver may compensate for the reduced risk through behaviours such as driving faster and overtaking more. Whether, or not, vision enhancement offers a valuable technological addition to improving driving performance is clearly an open debate. In a recent review of a book on Ergonomics and Safety of Intelligent Driver Interface edited by Noy (1997), Johnson (1998) was rather critical of the fact that the studies avoided (in his words) "the thorny issue of risk homeostasis theory." He was concerned that drivers might well compensate for the riskreducing technology through behavioural adjustments. This topic is the focus of the study reported in this paper.

Previous researchers have made convincing arguments that simulators offer a relevant medium for laboratory investigation of RHT (Jackson \& Blackman, 1994; Glendon et al. 1996, Heino et al. 1996) and the effects of advanced vehicle systems on driving behaviour (Stanton \& Young 1998). In particular, simulators enable researchers to 
Stanton, N. A. \& Pinto, M. (2000) Behavioural compensation by drivers of a simulator when using a vision enhancement system. Ergonomics 43, 1359-1370.

manipulate experimental variables and examine driver behaviour with a degree of control that is rarely possible in field studies. From a practical point of view, it is cheaper to develop a computer-based simulation than to manufacture the advanced technology for experimental purposes. Simulators also overcome ethical concerns about the safety of participants in the study as well as compressing experience (i.e. presenting a series of conditions that would not normally be experience with the same time frame). For an overview of the simulator debate, the reader is referred to Senders (1991) and Stanton (1996). It should be pointed out, however, that simulator-based research does not ordinarily allow the pathways of mode migration and avoidance to be explored.

\section{Method}

\subsection{Design}

The study used a completely repeated factorial design, in which all participants experienced all six driving conditions: daytime, night-time, night with vision enhancement, daytime with fog, daytime with fog and vision enhancement, daytime with fog and vision enhancement failure. Dependant variables included driver's speed and number of overtakes.

\subsection{Participants}

Eleven drivers (6 male and 5 female) participated in the study, they had a mean age of 21 years. All participants were undergraduates at the University of Southampton and met the acceptance criteria of a minimum of two years driving experience (all holders of current UK driving licences). Average driving mileage for the participants was approximately 10,000 miles.

Ethical permission was sought and granted by the Department of Psychology at the University of Southampton. In accordance with BPS and APA guidelines, all participants were made aware of their right to withdraw from the study at any time and were provided with a full debriefing about the aims of the study following their participation. 
Stanton, N. A. \& Pinto, M. (2000) Behavioural compensation by drivers of a simulator when using a vision enhancement system. Ergonomics 43, 1359-1370.

\subsection{Equipment}

The study was conducted in the Southampton Driving Simulator. The simulator comprises the front half of a Ford Orion registered in 1993 in which the participant sits in the drivers seat. The front portion of the Ford Orion is identical to the Ford Escort. The vehicle is fitted with transducers to the main vehicle inputs, an Acorn Archimedes RISC PC (running bespoke driving simulation software taking data from driver inputs via accelerator, brake and steering wheel), and an Epson LCD colour projection monitor (which displays the road view and the position of other vehicles to the participant on a screen in front of the car). Data automatically recorded every 0.5 seconds include vehicle speed, distance to vehicle in front, lateral position on the road, position of the steering wheel, positions of the brake and accelerator. The simulator is further described in journals papers resulting from other studies (see Stanton at al. 1997, Stanton \& Young 1998, Young \& Stanton 1997). These previous studies have shown that only three of the dependent measures are worthy of consideration: speed, distance to vehicle in front and lateral position on the road. Overtaking behaviour was calculated from the latter of these two measures. Position of the steering wheel, brake and accelerator are meaningless without considering the context of the behaviour whereas the first three measures provide an equitable basis for comparing the experimental groups.

The simulation also allowed driving conditions to be manipulated, specifically a daytime mode, a night-time mode and a fog mode. The vision enhancement system was activated by pressing a button on the dashboard. It could be deactivated by pressing the same button for a second time. The activated vision enhancement system was simulated by presenting a red (simulating an infra-red display) overlay projected head-up on the drivers forward field-of-view. Failure of the vision enhancement system was simulated by 'white-out' on the display. Under such circumstances the driver is advised to turn the system off by pressing the button on the dashboard. Whilst it is accepted that this is a simulation of the vision enhancement system, it is a reasonable representation of how a prototype device functions.

\subsection{Procedure}

Prior to participating in the study, participants were asked about their driving experience, to see if they met the minimum criterion, and data collected on age an annual mileage. On meeting the criterion, participants were briefly told that the nature of the study was to conduct an investigation into the operation of a vision enhancement system. They familiarised themselves with the controls of the car, were asked to drive in the same manner that they would in their own car, and were told that they would 
Stanton, N. A. \& Pinto, M. (2000) Behavioural compensation by drivers of a simulator when using a vision enhancement system. Ergonomics 43, 1359-1370.

encounter simulated night and fog driving conditions. A trial run of driving was given for 10 minutes so that the participants could get used to the feel of driving in the simulator. The experimental phase of the study was split into six runs of the driving simulator: day-time driving, night-time driving, vision-enhanced night-time driving, driving in foggy conditions, driving in foggy conditions with vision-enhancement, driving in foggy conditons after failure of the vision-enhancement system. The density of the fog represented a visibility range of 50 metres. The first run acted a control condition, followed by five experimental conditions, each lasting for 10 minutes. The justification for a fixed order of presenting the experimental conditions is based on the need to test the behavioural compensation hypothesis. The predicted behavioural compensation for increased vision requires that the conditions without vision enhancement are presented first. Similarly the failure scenario had to be presented last so that it would not confound the other experimental conditions. In the final experimental run, the vision enhancement system failed (effectively blanking out the field-of-view through the head-up, infra-red, display), requiring the participants to deactivate the system. Then participants were requested to reactivate the vision enhancement system.

\section{Results}

Two dependant variables were considered to be indicative of risk-taking behaviour for the purposes of this study: speed and overtaking. Mean speed (see figure 4) was calculated for each of the five driving conditions: daytime (Day), night-time (Night), night with vision enhancement (EV Night), daytime with fog (Fog), daytime with fog and vision enhancement (EV Fog).

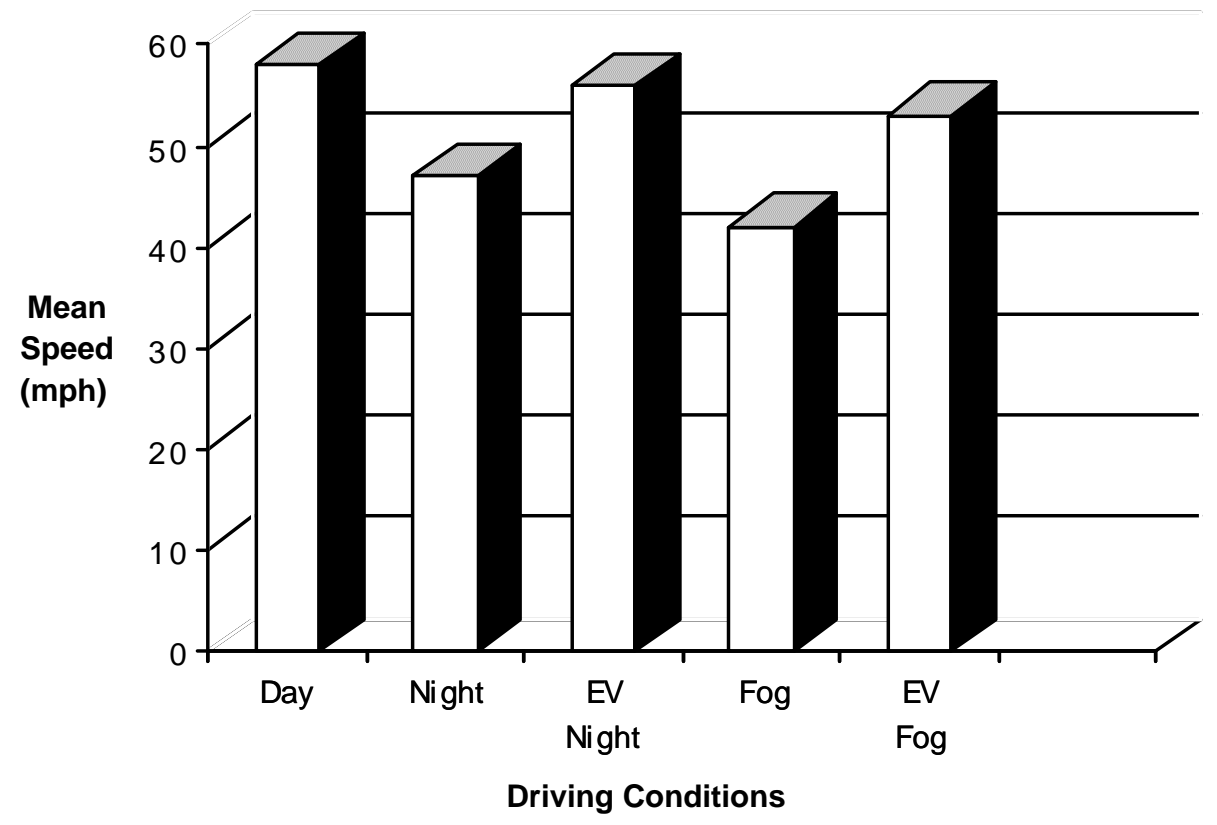


Stanton, N. A. \& Pinto, M. (2000) Behavioural compensation by drivers of a simulator when using a vision enhancement system. Ergonomics 43, 1359-1370.

Figure 4. Mean driver's speed under experimental driving conditions.

As figure 4 illustrates, there are statistically significant differences in driving speed associated with changes in the driving conditions $(\mathrm{F}(4,40)=9.31, \mathrm{p}<0.001)$. Participant's speed was lower in night and fog conditions than in day-time driving. Driving speed was significantly faster in the fog and night driving conditions with vision enhancement than those without $(\mathrm{F}(1,10)=12.48, \mathrm{p}<0.01)$. There were no statistical differences between the speeds in the Night and Fog conditions $(F(1,10)=3.67, p=N S)$, nor were there any statistical differences between the speeds in the vision enhanced conditions $(\mathrm{F}(1,10)=0.87, \mathrm{p}=\mathrm{NS})$. In line with the behavioural compensation hypothesis, these data suggest that participant's compensate for the changes in the level of environmental risk by adjusting their speed accordingly. This hypothesis was further supported by comparing driving speed with the vision enhancement (VE) before and after failure of the system. The means are plotted in figure 5 together with day-time and fog driving speeds.

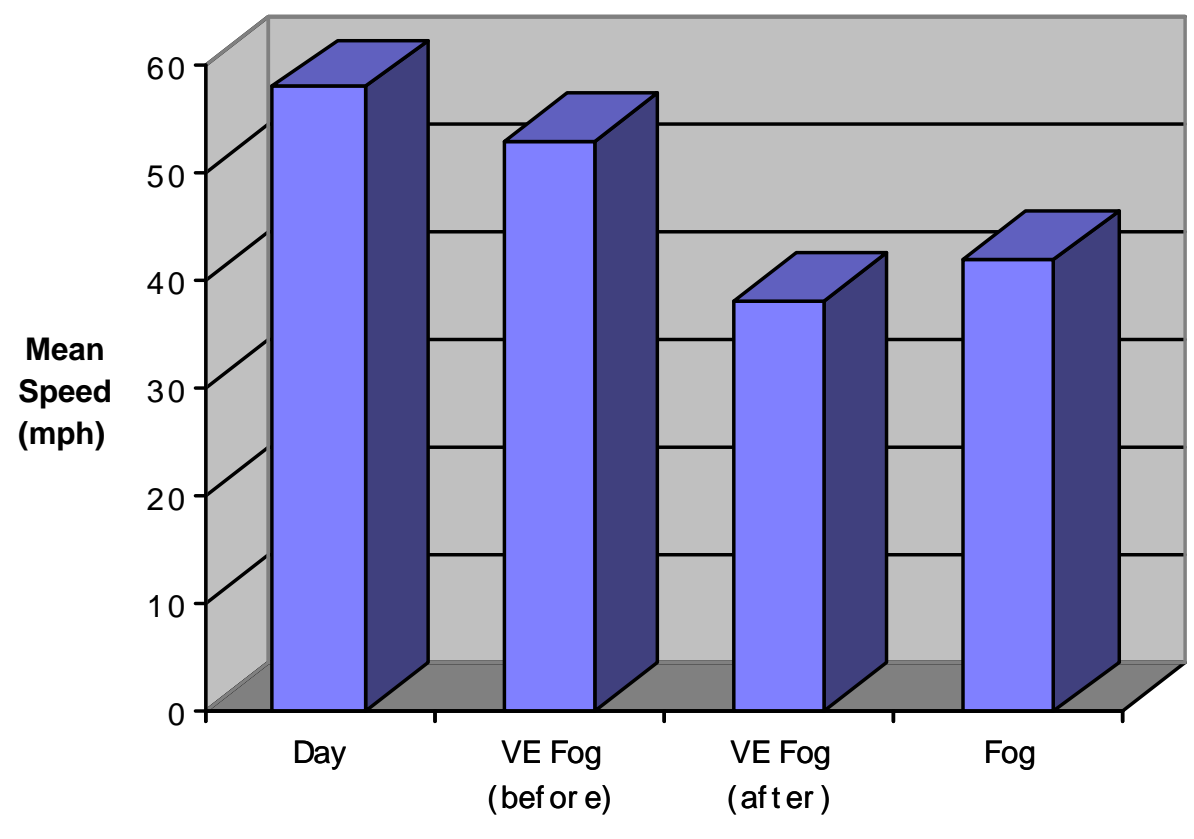

Driving Conditions

Figure 5. Comparing driving speed before and after VE failure.

As figure 5 indicates, the driving speed before Vision Enhancement (VE) failure was closer to day-time driving, whereas the driving speed after Vision Enhancement failure was closer to fog driving. There were statistically significant differences in driving speed before and after Vision Enhancement failure $(T=4.16, p<0.001)$ even though the Vision Enhancement system was activated in both conditions, and restored in the VE 
Stanton, N. A. \& Pinto, M. (2000) Behavioural compensation by drivers of a simulator when using a vision enhancement system. Ergonomics 43, 1359-1370.

Fog (after) condition. This lends further support to the behavioural compensation hypothesis, because not only do participants change their driving speed with the introduction of vision enhancement (i.e. equating increased vision with decreased risk), they also change their speed when their perception of environmental risk is changed (i.e. the failure of the VE system requires a re-examination of the first hypothesis). There even seems to be some suggestion of over-compensation, if the mean speeds for fog-driving and VE Fog (after) conditions are compared in figure 5.

As well as assessing driving speed, the number of overtakes were determined to see if participants's behaviour changed with the activation of the Vision Enhancement system. The mean number of overtakes per driving minute are presented in figure 6 for each of the five driving conditions: daytime (Day), night-time (Night), night with vision enhancement (EV Night), daytime with fog (Fog), daytime with fog and vision enhancement (EV Fog).

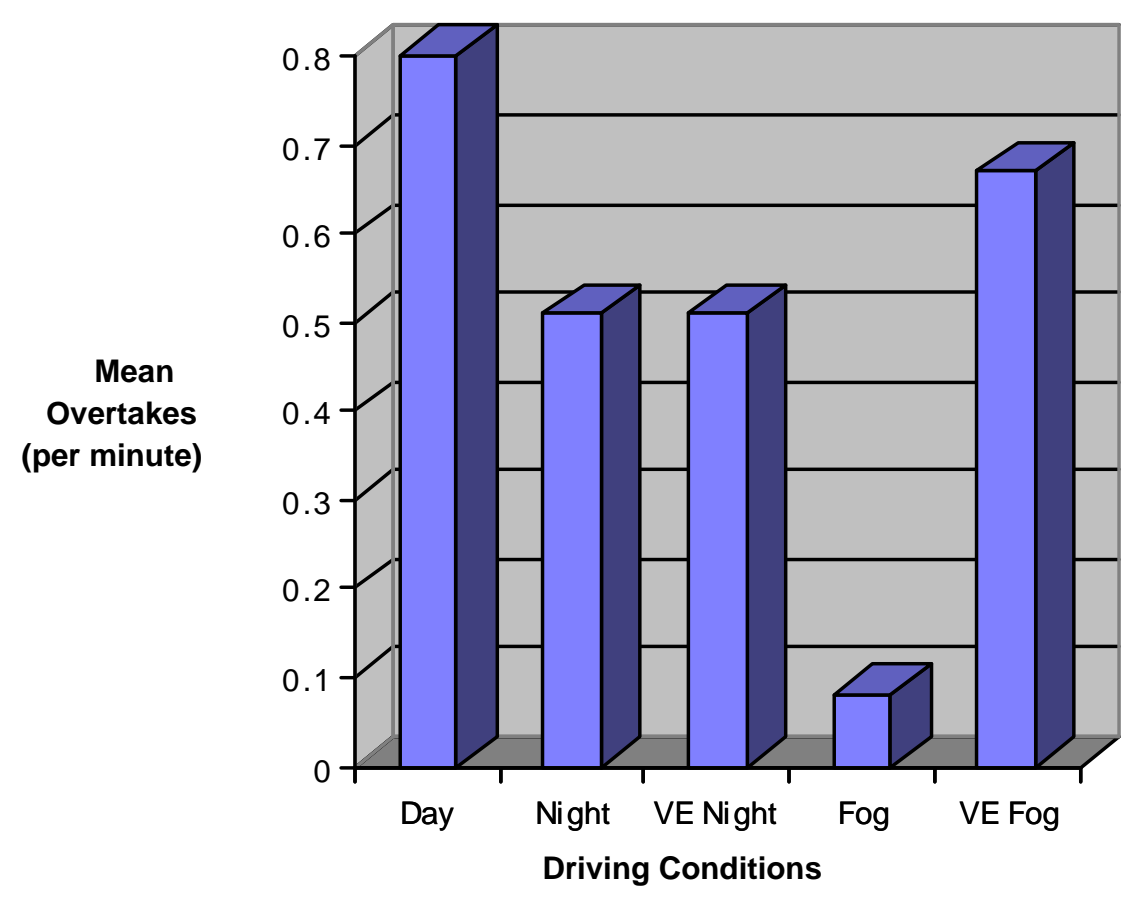

Figure 6. Mean number of overtakes under experimental driving conditions.

As with driving speed, there were statistically significant differences in the number of overtakes between the driving conditions $(\mathrm{F}(4,40)=8.07, \mathrm{p}<0.001)$. As could be expected, most overtakes took place in the day-time driving condition and least in the 
Stanton, N. A. \& Pinto, M. (2000) Behavioural compensation by drivers of a simulator when using a vision enhancement system. Ergonomics 43, 1359-1370.

fog driving condition. Vision Enhancement appears to have had little effect on night driving, whereas it has had a much larger effect on fog driving, illustrated statistically by an interaction between weather and vision factors $(F(1,10)=7.57, p<0.05)$. The differential effects between night and fog driving are noted, and will be discussed later.

\section{Discussion}

As shown in the results section, participants in this study drove faster during the day condition than in the fog and night driving conditions. This is consistent with the effects of the reduced visibility placing constraints on the driver, requiring them to reduce their speed in order to maintain safe driving. The activation of the Vision Enhancement system in the reduced visibility condition (i.e. fog and night driving) was accompanied by an increase in driving speed. Effective use of Vision Enhancement affords drivers greater visibility in adverse driving conditions and enables them to driver at faster speeds than ordinarily possible. The findings also accord well with RHT, and in particular the behavioural compensation hypothesis: the reduction in environmental risk associated with the activation of the Vision Enhancement system leads to an increase in driver speed. The net result being that the level of risk is restored to previous levels, providing that the Vision Enhancement system performs reliably.

When drivers have been supplied with new information, however, about the level of intrinsic risk (i.e. the potential of the Vision Enhancement system to fail), they reassessed their utilities (i.e. the costs and benefits associated with cautious and risky behaviours) and adjusted their behaviour accordingly to restore the desired level of target risk. It is not claimed that any of this reassessment occurred overtly, the supposition, based on RHT, is used to explain the changes in driving behaviour. In this case, when drivers reduced their speed when using the Vision Enhancement system in fog to speeds similar to fog-diving without Vision Enhancement, following a catastrophic failure of the system. Simulations of this kind are particularly important when thinking about the introduction of new technology. This is never moresohen faced with potentially life-threatening consequences in certain operational scenarios when thinking about the introduction of new technology.

Informal observation of the participants at the time that the Vision Enhancement system failed, showed a moment of indecisiveness followed by a search for the button to deactivate the system. After deactivating the system, participants were asked to reactivate it. This procedure led to the discovery of the compensatory behaviour. Muir and Moray (1996) have shown that once people develop distrust in technological systems, they find it difficult to regain the previous levels of trust. They also argue that 
Stanton, N. A. \& Pinto, M. (2000) Behavioural compensation by drivers of a simulator when using a vision enhancement system. Ergonomics 43, 1359-1370.

performance of human-machine systems is better with some level of distrust by the human operator, as this increases sampling behaviour. The study reported in this paper could be extended by considering behaviour after the VE system failure in more details, to consider (i) how long the behavioural compensation effect lasts, (ii) does the behavioural compensation effect transfer to other VE conditions, such as night driving, and (iii) does the effect transfer to non-VE conditions, i.e other situations where a driver puts their trust in new technology. Stanton and Young (1998) have proposed that trust together with locus-of-control, stress, workload, feedback and situational awareness are important psychological concepts when attempting to understand the implications of the use of new technology in vehicles.

In another study looking at behavioural compensation and limited visibility, Ward and Wilde (1966) predicted that car drivers speed would increase if they had a clearer view on the approach to a railway crossing. To test the theory in a field study, Ward and Wilde compared driver behaviour at two railway crossing sites. The control site was not modified and had an obscured view of the trains' approach path whereas the modified site had a clear view of the trains' approach path. Ward and Wilde showed that driver's speed was greater at the site with better visibility, as a result of which no net safety benefit was realized. Thus the findings from Ward and Wilde are very similar to those reported in this paper: drivers compensate for better visibility by driving faster. There are many other studies to show behavioural compensation in a variety of ways (e.g. Glendon, et al. 1996, Hoyes et al. 1996).

In summary, there are some important findings in this study. First, the vision enhancement system enables drivers to continue to drive at near day-time driving levels under foggy conditions. This, however, has the effect of restoring the level of risk to pre-existing levels. Second, simulated failure of the Vision Enhancement system leads to a reduction in driving speed, lending further support to RHT. This has enabled us to assess how drivers might behave in a failure scenario. Third, the usefulness of simulators has been demonstrated for conducting studies of advanced vehicle technology and compressing experience within an ethical research paradigm. This enables researchers to test the effects of potential changes in the driving environment and assess the effects on the driver in an efficient manner.

What this study has not addressed is the effects of a vehicle driving with the Vision Enhancement system in conditions of reduced visibility (e.g. fog) on the same stretch of highway as other vehicles that do not have the capability of Vision Enhancement. Potentially, the Vision Enhancement equipped vehicle could become a hazard to other 
Stanton, N. A. \& Pinto, M. (2000) Behavioural compensation by drivers of a simulator when using a vision enhancement system. Ergonomics 43, 1359-1370.

road users, if the driver drove in a way inconsistent with the road conditions. One of our participants commented that they were unsure if it was safe to overtake another car in the fog. In this particular situation, the car ahead was moving very slowly around a corner, and even though the participant could see the road ahead was clear for the overtake, he was unsure of the effect on the other driver. He reasoned that the driver without the benefit of Vision Enhancement, who was suddenly overtaken at speed by another driver, might react inappropriately through fright or surprize. 
Stanton, N. A. \& Pinto, M. (2000) Behavioural compensation by drivers of a simulator when using a vision enhancement system. Ergonomics 43, 1359-1370.

\section{Conclusions}

As Trimpop (1996) suggests, the weight of evidence tips in favour of RHT, but this is problem for vehicle manufacturers wishing to make driving safer. The introduction of greater measures of environmental safety, such as Vision Enhancement systems, may only be compensated for by drivers. Stanton \& Glendon (1996) argue that personcentred approaches are likely to be more successful than technical solutions alone. The engineering disciplines are good at producing technology that has great potential to make driving safer, but just placing the technology in the vehicle will not necessarily mean that this potential is realised. Indeed, analysis of accident statistics shows a shortfall in expected benefits from the introduction of safety mechanisms and experimental studies conducted in simulators seem to lend support to RHT. The challenge for psychologists and ergonomists is to find ways in which this technology can be used to make driving safer. If drivers could be persuaded to drive in the manner exhibited after the failure of the vision enhancement system, then the introduction of such as system would greatly improve traffic safety. Thus the challenge for ergonomics may be defined as reminding drivers of a safe driving speed in the fog with the vision enhancement system engaged, without having to resort to periodic system failure. There are at least two possible solutions, passive or active. An example of a passive solution might be to suggest a safe driving speed to the driver, based upon fog density and road condition, via an in-vehicle information system. An example of an active solution might be change the vision enhancement, head-up, display dynamically. For instance, if the driver's speed exceeds the recommended speed, then the distance ahead displayed by the vision enhancement system is reduced correspondingly. In this way, the drivers cannot use the vision enhancement system to driver faster in foggy condition, because the system will compensate by reducing headway visibility. The immediacy of feedback to the driver should, in all probability, coerce the appropriate driving behaviour. From a psychological perspective, compensation effects observed in many RHT studies may be interpreted as reflecting individuals' attempts to maximize the degree of mastery or control over their environment. Only by anticipating the likely effects of driving technology on the driver, and conducting empirical research in simulated environments, can an understanding be gained on how to make transportation systems safer. 
Stanton, N. A. \& Pinto, M. (2000) Behavioural compensation by drivers of a simulator when using a vision enhancement system. Ergonomics 43, 1359-1370.

\section{Acknowledgement}

This paper was written whilst the first author was on sabbatical leave as a visiting fellow at Cornell University in the USA.

\section{References}

DINGUS, T. A.; HULSE, M. C.; MOLLENHAUSER, M. A.; FLEISCHMAN, R. N.; MCGEHEE, D. V. AND MANAKKAL, N. 1997, a Effects of age, system experience, and navigation techniques on driving with an advanced traveller information system. Human Factors, 39, 177-199.

DINGUS, T. A.; MCGEHEE, D. V; MANAKKAL, N.; JAHNS, S. K.; CARNEY, C. AND HANKEY, J. M. 1997, b Human factors field evaluation of automotive headway maintenance/collision warning devices. Human Factors, 39, 216-229.

GLENDON, A. I.; HOYES, T. W.; HAIGNEY, D. E. AND TAYLOR, R. G. 1996 A review of risk homeostasis theory in simulated environments. Safety Science, 22, 15-25.

HEINO, A.; VAN DER MOLEN, H. H. AND WILDE, G. J. S. 1996 Risk perception, risk taking, accident involvement and the need for simulation. Safety Science, 22, 35-48.

HOYES, T. W.; STANTON, N. A. AND TAYLOR, R. G. 1996 Risk homeostasis theory: a study of intrinsic compensation. Safety Science, 22 (1-3), 77-86.

HOYOS, C. G. 1988 Mental load and risk in traffic behaviour. Ergonomics, 31, 571-584.

JACKSON, J. S. H. AND BLACKMAN, R. 1994 A driving-simulator test of Wilde's risk homeostasis theory. Journal of Applied Psychology, 78, 950-958.

JACKSON, P. G. 1988 In search of better route guidance instructions. Ergonomics, 41, 1000-1013.

JOHNSON, G. 1998 Book review: Ergonomics and safety of intelligent driver interfaces edited by Y. I Noy. New Jersey: Lawrence Erlbaum (1997). Ergonomics, 41 (7), 10821083

MCKENNA, F. P. 1988 What role should the concept of risk play in theories of accident involvement? Ergonomics, 31, 469-481. 
Stanton, N. A. \& Pinto, M. (2000) Behavioural compensation by drivers of a simulator when using a vision enhancement system. Ergonomics 43, 1359-1370.

MUIR, B. M. \& MORAY, N. 1996 Trust in automation: Part II. Experimental studies of trust and human intervention in process control simulation. Ergonomics. 39, 429-460.

NILSSON, L. 1995 Safety effects of adaptive cruise control in critical traffic situations. Proceedings of the Second World Congress on Intelligent Transport Systems: "Steps Forward", Volume III: Yokohama, VERTIS. 1254-1259.

NOY, Y. I. 1997 ed., Ergonomics and Safety of Intelligent Driver Interfaces. New Jersey: Erlbaum.

SENDERS, A. F. 1991 Simulation as a tool in the measurement of human performance. Ergonomics, 34, 995-1025.

SRINIVASAN, R AND JOVANIS, P. P. 1997 Effect of selected in-vehicle route guidance systems on driver reaction times. Human Factors, 39, 200-215.

STANTON, N. A. 1996 Simulators: research and practice, in: N. A. Stanton (Ed) Human Factors in Nuclear Safety. Taylor and Francis, London, 117-140.

STANTON, N. A. AND GLENDON, A. I. 1996 Risk homeostasis and risk assessment. Safety Science, 22, 1-13.

STANTON, N. A. AND MARSDEN, P. 1996 From fly-by-wire to drive-by-wire: safety implications of vehicle automation. Safety Science, 24, 35-49.

STANTON, N. A. \& YOUNG, M. S. 1998 Vehicle automation and driving performance. Ergonomics, 41, 1014-1028.

STANTON, N. A.; YOUNG, M. \& MCCAULDER, B. 1997 Drive-by-wire: the case of driver workload and reclaiming control with Adaptive Cruise Control. Safety Science, 27, 149-159.

TRIMPOP, R. M. 1996 Risk homeostasis theory: problems of the past and promises for the future. Safety Science, 22, 119-130.

WARD, N. J. AND WILDE, G. J. S. 1996 Driver approach behaviour at an unprotected railway crossing before and after enhancement of lateral sight distances: an 
Stanton, N. A. \& Pinto, M. (2000) Behavioural compensation by drivers of a simulator when using a vision enhancement system. Ergonomics 43, 1359-1370.

experimental investigation of a risk perception and behavioural compensation hypothesis. Safety Science, 22, 63-75.

WILDE, G. J. S. 1982 The theory of risk homeostasis: implications for safety and health. Risk Analysis, 2, 209-225.

WILDE, G. J. S. (1994) Target Risk. Toronto, PDE Publications.

YOUNG, M. \& STANTON, N. A. (1997) Automotive automation: Implications for driver mental workload. International Journal of Cognitive Ergonomics, 1, 325-336. 
Stanton, N. A. \& Pinto, M. (2000) Behavioural compensation by drivers of a simulator when using a vision enhancement system. Ergonomics 43, 1359-1370.

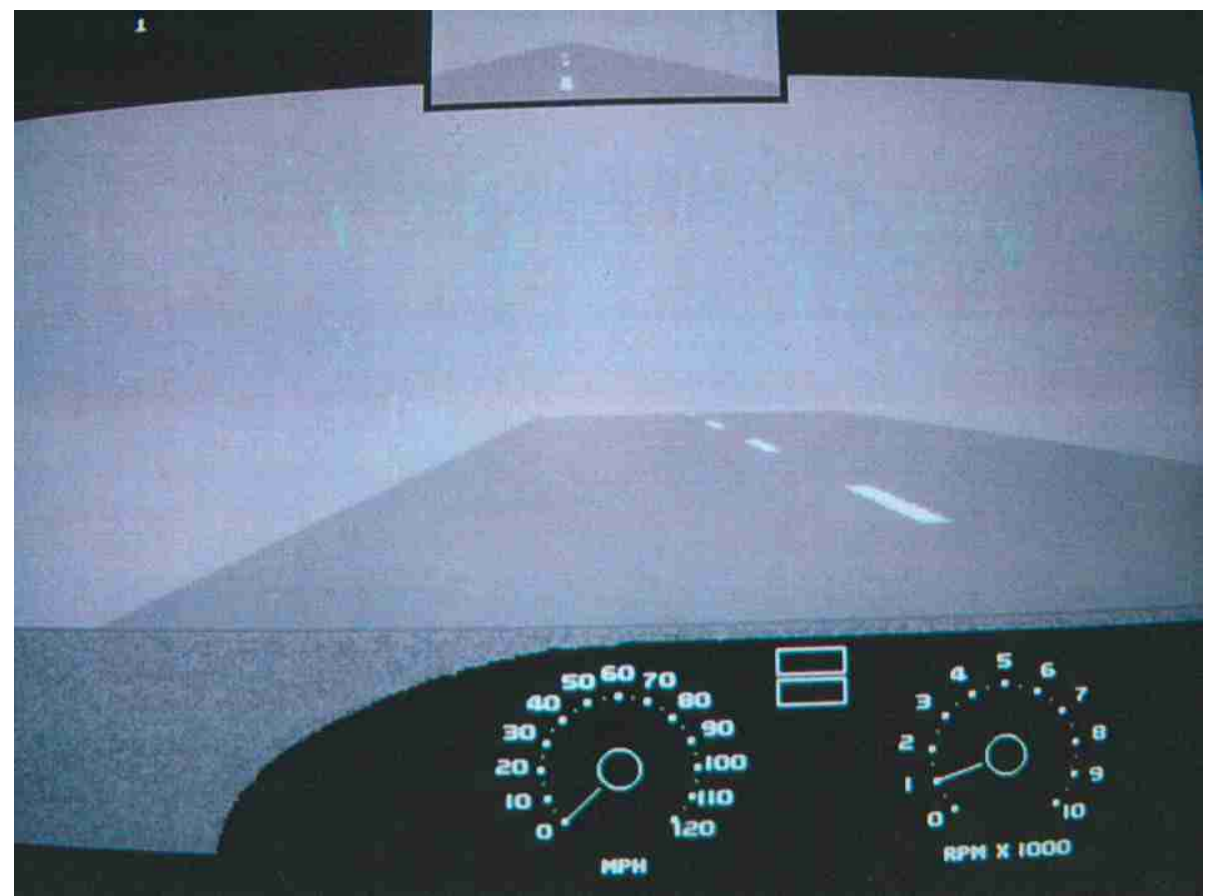

Figure 1. Driving in fog without vision enhancement. 
Stanton, N. A. \& Pinto, M. (2000) Behavioural compensation by drivers of a simulator when using a vision enhancement system. Ergonomics 43, 1359-1370.

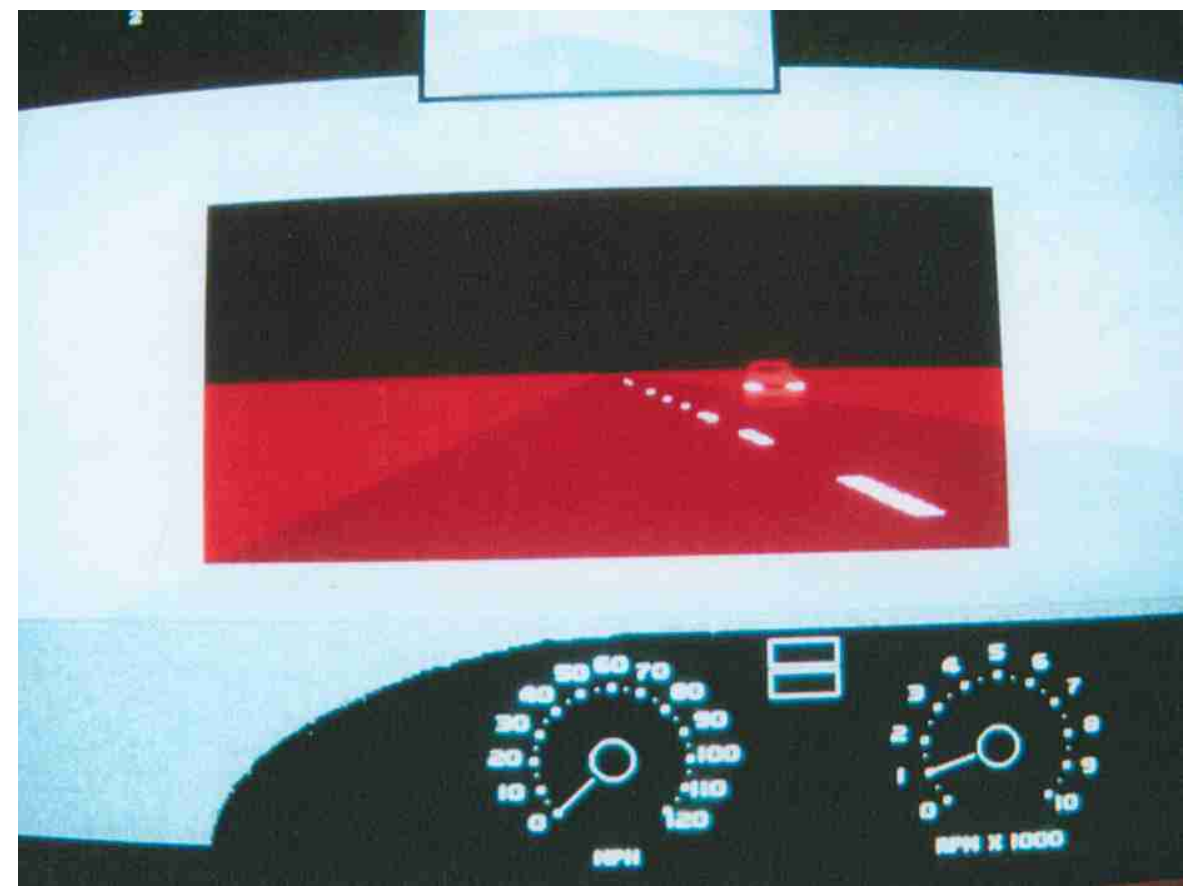

Figure 2. Driving in fog with vision enhancement. 
Stanton, N. A. \& Pinto, M. (2000) Behavioural compensation by drivers of a simulator when using a vision enhancement system. Ergonomics 43, 1359-1370.

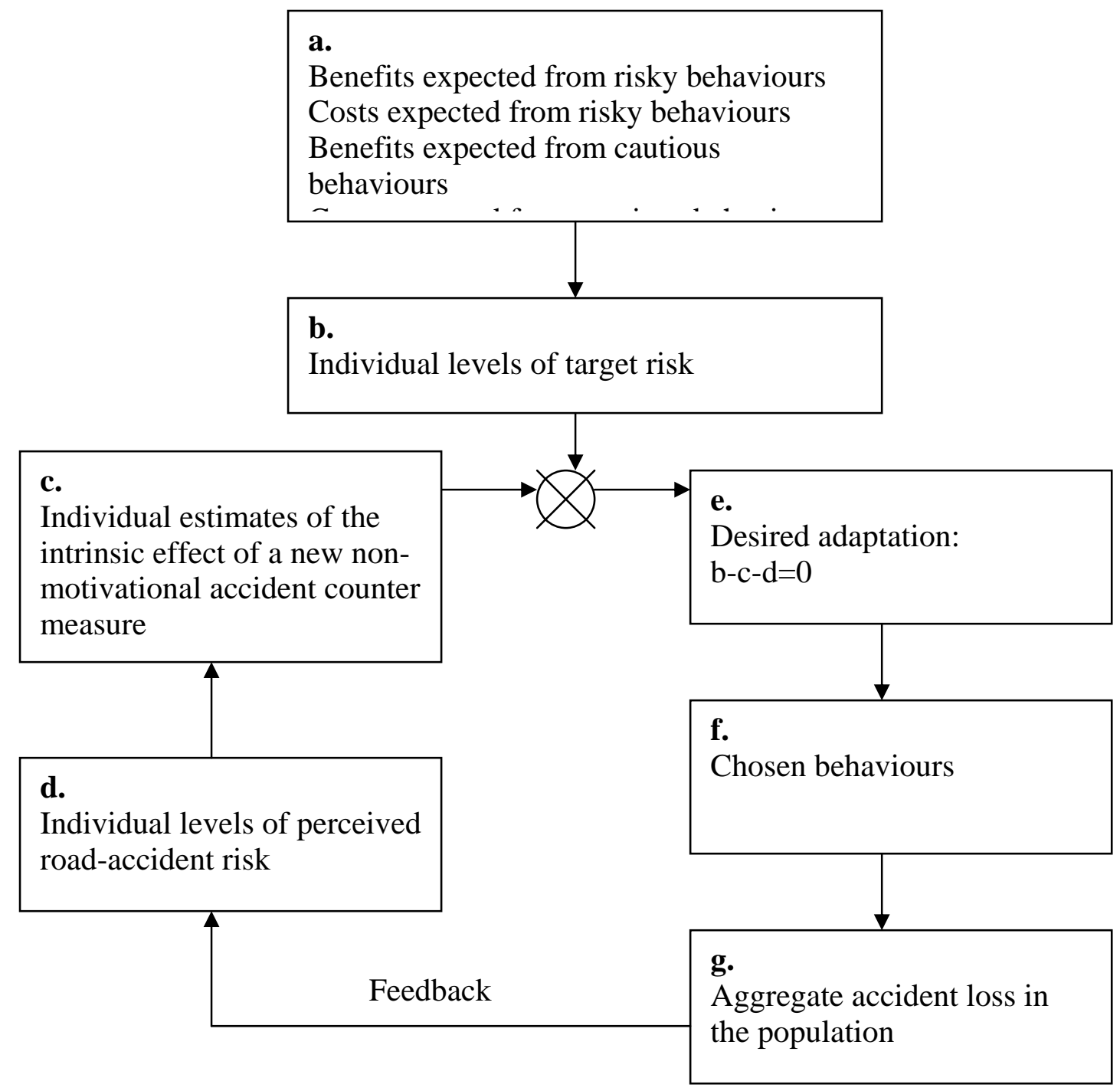

Figure 3. Risk homeostasis theory 
Stanton, N. A. \& Pinto, M. (2000) Behavioural compensation by drivers of a simulator when using a vision enhancement system. Ergonomics 43, 1359-1370.

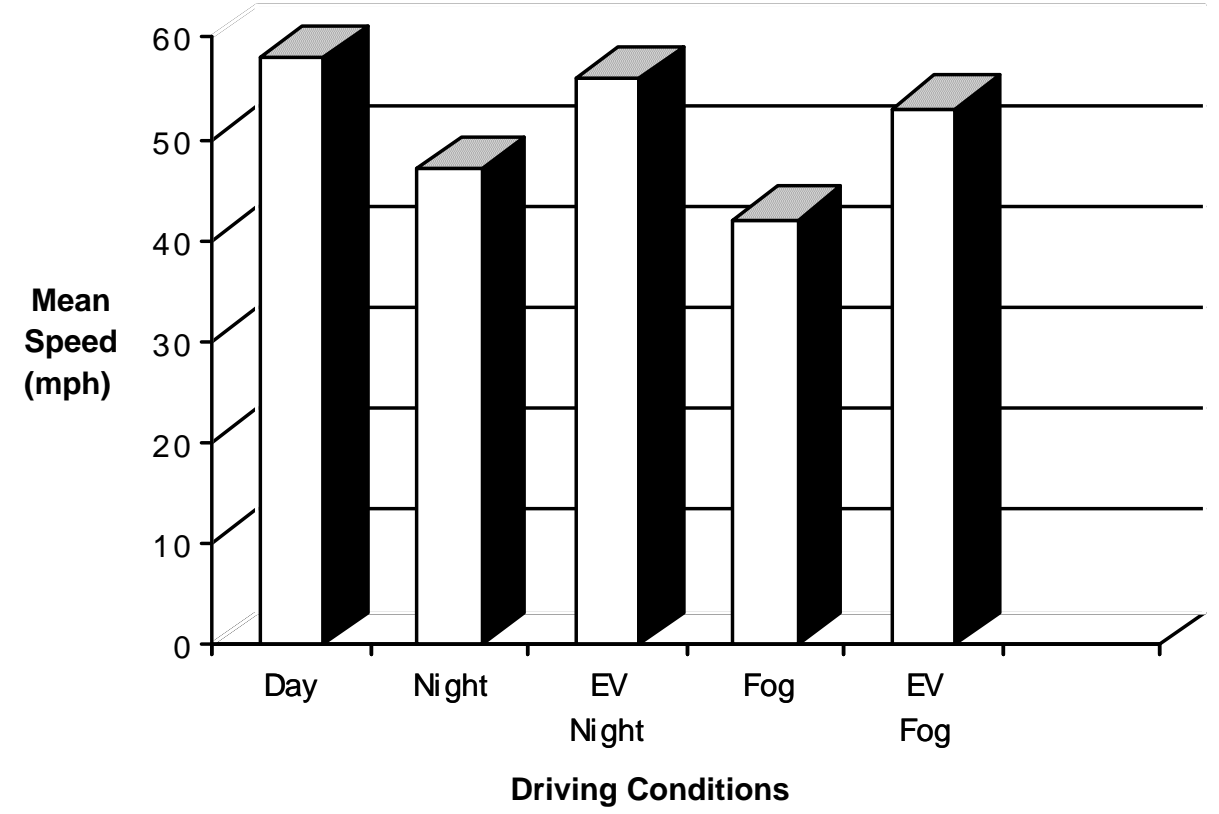

Figure 4. Mean driver's speed under experimental driving conditions. 
Stanton, N. A. \& Pinto, M. (2000) Behavioural compensation by drivers of a simulator when using a vision enhancement system. Ergonomics 43, 1359-1370.

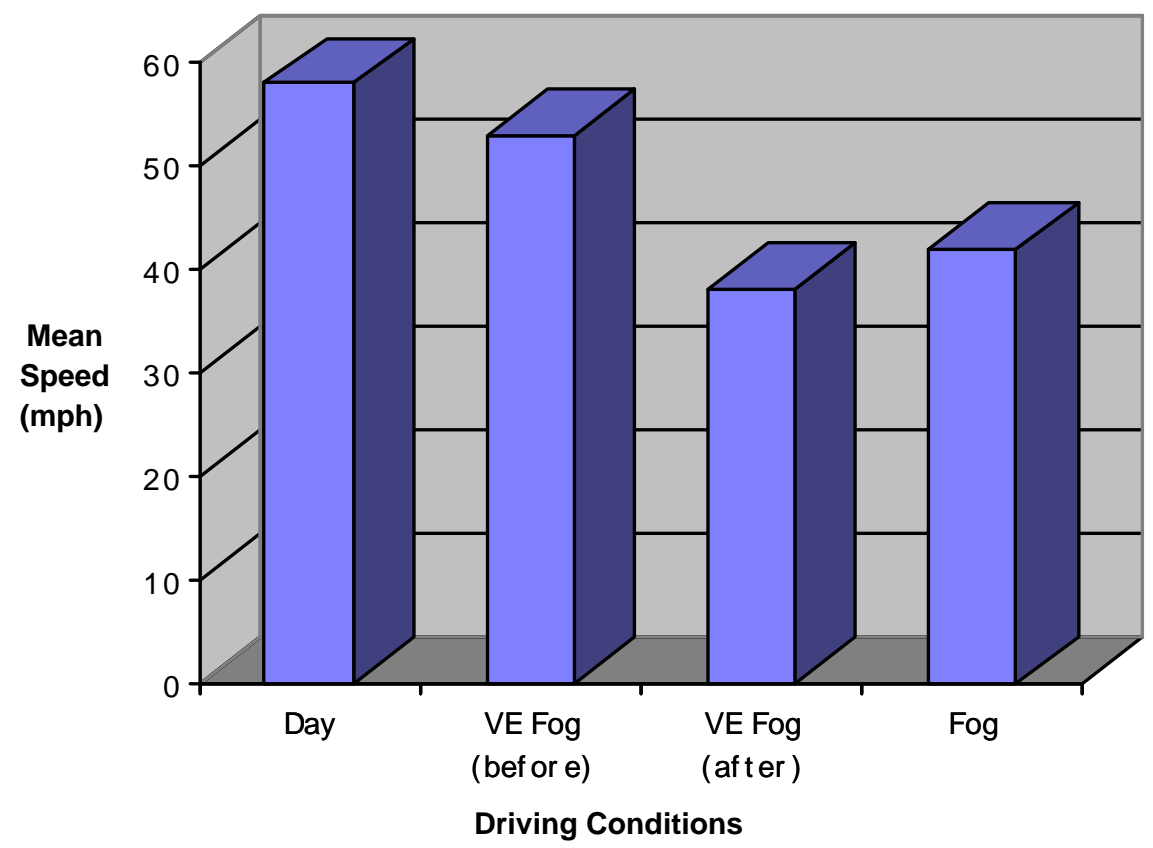

Figure 5. Comparing driving speed before and after VE failure. 
Stanton, N. A. \& Pinto, M. (2000) Behavioural compensation by drivers of a simulator when using a vision enhancement system. Ergonomics 43, 1359-1370.

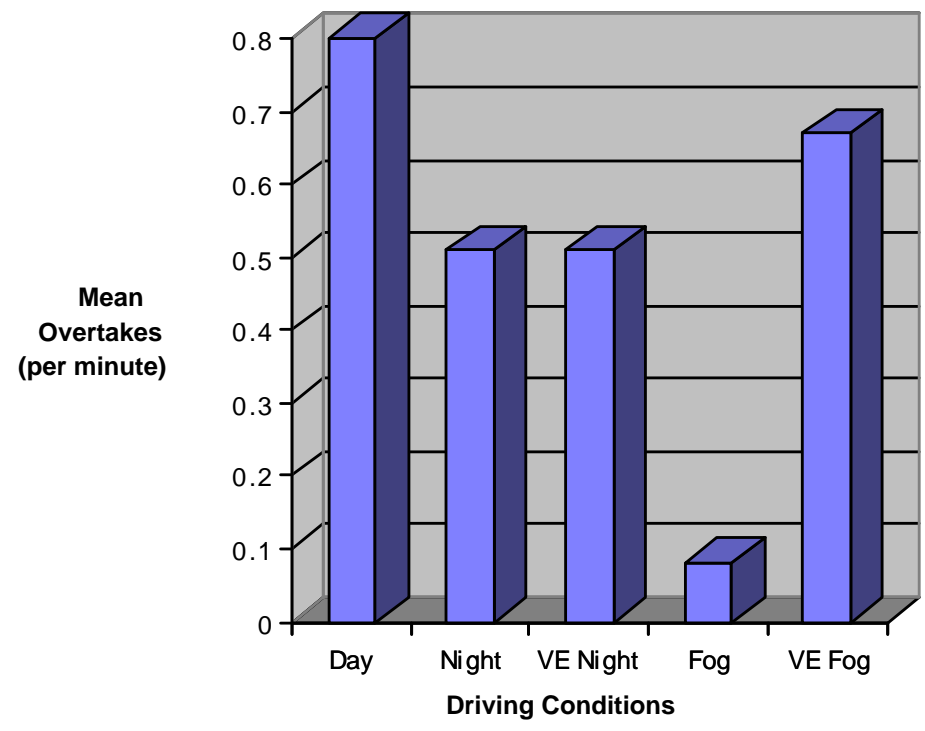

Figure 6. Mean number of overtakes under experimental driving conditions. 